Introduction: The adrenal gland is a frequent site of metastases in different types of cancer. The aim of this study was to assess the results of metastatic adrenalectomy in a single institution and to identify factors for survival.

Material and methods: A retrospective, single-centre analysis of outcomes of 39 patients (22 male, 17 female) with adrenal metastases who underwent surgery within 14 years (20042017) was performed. The median age at the time of adrenal surgery was 64.8 years (range 49-79 years).

Results: In our study group non-small cell lung cancer (NSCLC) was the most frequent primary tumour type (15 pts), followed by renal cell carcinoma (RCC) (14 pts) and colon cancer (6 pts). Most of the metastases - 36 (92\%) - were metachronous (> 6 months). All synchronous metastases were NSCLC. The mean time from primary cancer to adrenalectomy was 42.3 months (range 1-176) and was statistically longer for RCC. In 3 patients (8\%) metastases were bilateral and both adrenal glands were removed. In all patients, surgery was limited to the adrenal gland, and no major complications of surgery were observed. The median overall survival after metastasectomy was 18 months (3-81) and was statistically longer for colon cancer -29.5 months $(p=0.012)$. In patients who died, tumours were significantly bigger than in survivors, $76.5 \mathrm{~mm}$ vs. $52.5 \mathrm{~mm}$ $(p=0.026)$.

Conclusions: Surgery for adrenal metastasis is safe and indications for this procedure should be individualized. In selected patients, surgical removal of adrenal metastasis was associated with longer survival.

Key words: adrenalectomy, adrenal metastases, adrenal mass, median overall survival.

Contemp Oncol (Pozn) 2020; 24 (1): 29-33 DOI: https://doi.org/10.5114/wo.2020.93679

\section{Adrenal metastases - long-term results of surgical treatment, single-centre experience}

\author{
Radosław Samsel ${ }^{1}$, Andrzej Cichocki ${ }^{1}$, Katarzyna Roszkowska-Purska ${ }^{2}$, \\ Lucyna Papierska3 ${ }^{3}$ Agnieszka Koalasińska-Ćwikła ${ }^{4}$, Edyta Karpeta ${ }^{5,6}$, \\ Tomasz Ostrowski ${ }^{1}$, Karolina Nowak ${ }^{3}$
}

${ }^{1}$ Department of Surgery, Clinic of Oncological Surgery and Neuroendocrine Tumors, Maria Sklodowska-Curie Institute - Oncology Center, Warsaw, Poland

${ }^{2}$ Department of Pathology, Maria Sklodowska-Curie Institute - Oncology Center, Warsaw, Poland

${ }^{3}$ Department of Endocrinology, Centre of Postgraduate Medical Education,

Bielański Hospital, Warsaw, Poland

${ }^{4}$ Department of Oncology and Radiotherapy, Maria Sklodowska-Curie Institute Oncology Center, Warsaw, Poland

${ }^{5}$ Department of General and Transplant Surgery, Infant Jesus Teaching Hospital, Warsaw, Poland

${ }^{6}$ Department of Surgical and Transplantation Nursing and Extracorporeal Therapies, Medical University of Warsaw, Warsaw, Poland

\section{Introduction}

The most common types of adrenal tumours are benign cortical adenomas [1], although the adrenal gland is one of the most prevalent sites for metastases from various malignancies and metastases are the most frequent malignant tumours of the adrenal gland. The existence of adrenal metastases in patients with a history of cancer varies in different series from 10 to $27 \%[2,3]$. Usually, they are a part of disseminated disease while isolated adrenal metastases occur only in less than one percent of patients [4].

Surveillance protocols in treated cancer patients have increased the identification of incidental adrenal lesions, of which a high proportion are metastases. More reliable and sensitive methods of diagnostic imaging lead to earlier detection of adrenal metastases.

Selection of patients with adrenal metastases for curative treatment remains a big challenge.

Incidental adrenal masses are detected in approximately $4-5 \%$ of patients who undergo CT examinations. The occurrence of adrenal incidentalomas increases with age: from less than $0.5 \%$ of adrenal nodules revealed in patients in their 20 s compared with up to $7 \%$ in patients older than 70 years [5-8].

The majority of these tumours are benign nonfunctional lesions. On the other hand, the frequency of adrenal metastases is high. Opposite to these data, the number of adrenalectomies performed to treat metastases is low, from less than one performed in a surgical centre per year [9] up to less than six a year $[10,11]$. It is one of the reasons that makes it difficult to establish criteria to define which patients will benefit from adrenalectomy in terms of cure or extended survival. Published data are heterogeneous, differing in frequency of occurrence of tumours that metastasize to the adrenal glands.

Benefits of adrenalectomy for metastases are still unclear but several retrospective studies have identified highly selected groups of patients whose survival seems to be prolonged by surgery $[9,12,13]$.

We have assessed the results of metastatic adrenalectomy in our institution to identify prognostic factors for survival. 


\section{Material and methods}

This is a single-centre retrospective analysis of patients with adrenal metastases undergoing adrenalectomy in our hospital in which the final histopathologic report showed explicit evidence of adrenal metastatic disease. To identify patients with such diagnosis medical records were reviewed. Pre-operative clinical, radiological and biochemical data and detailed operation and histology reports were retrieved from patients' medical records, either in paper form or by electronic means. Hence, radiology and pathology reports were intentionally not re-evaluated, implying that the data were purely observational.

Over the period from January 2004 to December 2017, 430 patients with various adrenal tumours were referred to our centre for surgical treatment. In this group, 39 (9.1\%) patients with metastases to the adrenal gland were identified. Patients with a direct extension of primary tumour or renal cell carcinoma (RCC) with ipsilateral synchronous metastases were not included in the study as well as patients with suspicion of generalized disease. All other patients with isolated adrenal tumours suspected for malignancy were referred for surgery.

Metastases were defined as synchronous if detected within 6 months after primary surgery and those more than 6 months defined as metachronous. Disease-free interval (DFI) was the time between primary treatment and adrenalectomy.

The decision regarding operative treatment was based on diagnostic imaging. The risk of malignancy was determined by several factors including tumour size, radiographic features such as irregular margins, high density, slow washout of contrast medium, presence of necrosis, area of haemorrhage and calcifications. According to current guidelines, adrenal biopsies were not performed.

All patients were operated on under general anaesthesia with venous thromboembolism prophylaxis by low molecular weight heparins and appropriate antibiotic pro- phylaxis using a second-generation cephalosporin (cefuroxime) according to the hospital protocol.

For patients with large tumours or essential comorbidities, cross-matched blood was reserved. All patients were operated on by open surgery and the typical surgical approach was transabdominal lateral flank incision. In the presented period laparoscopic adrenalectomy was not used in patients with tumours suspected for malignancy.

21 left, 14 right and 4 bilateral, classical, open adrenalectomies were performed. There were no cases of mortality or major complications associated with surgery. The average time of hospital stay was 7 days (range 4-30). One prolonged hospitalization - 30 days - was related to wound pain requiring the use of opioids. The follow-up period was from 3 to 81 months.

Because most of the patients were referred for surgery from different oncological centres, systemic treatment was performed according to up-to-day protocols outside our centre and its analysis is not a part of this study.

Overall survival (OS) and disease-free survival (DFS) were estimated by the Kaplan-Meier method and differences in survival were compared by the log-rank test. Uni- and multivariable predictors of overall mortality were estimated by Cox regression analysis. A $p$ value of $<0.05$ was considered to be significant (Statistica ver. 13.1) (StatSoft, Poland).

\section{Results}

Thirty-nine patients with adrenal metastasis (17 female, 22 male), mean age 64.8 years (range 49-79 years), were treated by adrenalectomy in our centre during the study period. The diagnoses were: non-small cell lung cancer (NSCLC) - 15 cases, RCC - 14 cases, colorectal cancer - 6 cases and others (melanoma -2, ovarian -1, urothelial cancer -1) (Table 1).

The mean DFI between primary treatment and adrenal surgery for all patients was 42 months and range 1-179 (Table 2).

Table 1. Characteristics of tumours and patients

\begin{tabular}{lcccc}
$\begin{array}{l}\text { Type of primary } \\
\text { cancer }\end{array}$ & Number (\%) & Sex F/M & $\begin{array}{c}\text { Age at time of adrenal surgery (years) } \\
\text { Mean/range }\end{array}$ & $\begin{array}{c}\text { Size of metastatic tumour (mm) } \\
\text { Mean (range) }\end{array}$ \\
\hline NSCLC & $15(38.5)$ & $6 / 9$ & $64.5(49-79)$ & $67.2(15-130)$ \\
RCC & $14(36)$ & $6 / 8$ & $62.4(55-77)$ & $58.7(15-130)$ \\
Colon & $6(15.5)$ & $3 / 3$ & $66.2(57-70)$ & $67.4(30-105)$ \\
Others & $4(10)$ & $2 / 2$ & $71(65-78)$ & $69(55-95)$
\end{tabular}

NSCLC - non-small cell lung cancer, RCC - renal cell carcinoma

Table 2. Interval between primary treatment and adrenal surgery - group characteristics

\begin{tabular}{|c|c|c|c|c|c|c|}
\hline $\begin{array}{l}\text { Primary } \\
\text { tumour }\end{array}$ & $\begin{array}{l}\text { Time mean } \\
\text { (months) }\end{array}$ & $\begin{array}{c}\mathrm{Cl} \\
-95.000 \%\end{array}$ & $\begin{array}{c}\mathrm{Cl} \\
+95.000 \mathrm{v}\end{array}$ & $\begin{array}{c}\text { Time mean } \\
\text { (months) } \\
\text { SD }\end{array}$ & $\begin{array}{c}\text { Time } \\
\text { (months) } \\
\text { Min }\end{array}$ & $\begin{array}{c}\text { Time } \\
\text { (months) } \\
\text { Max }\end{array}$ \\
\hline NSCLC & 17.8 & 6.51240 & 29.0876 & 20.4 & 1.0 & 81.0 \\
\hline $\mathrm{RCC}$ & 81.6 & 49.69018 & 113.5098 & 57.6 & 6.0 & 176.0 \\
\hline Colon & 17.3 & 9.86340 & 24.8033 & 7.1 & 9.0 & 27.0 \\
\hline Others & 18.7 & -1.41238 & 38.7457 & 8.1 & 10.0 & 26.0 \\
\hline All & 42.3 & 26.53380 & 58.1329 & 48.7 & 1.0 & 176.0 \\
\hline
\end{tabular}

NSCLC - non-small cell lung cancer, RCC - renal cell carcinoma 


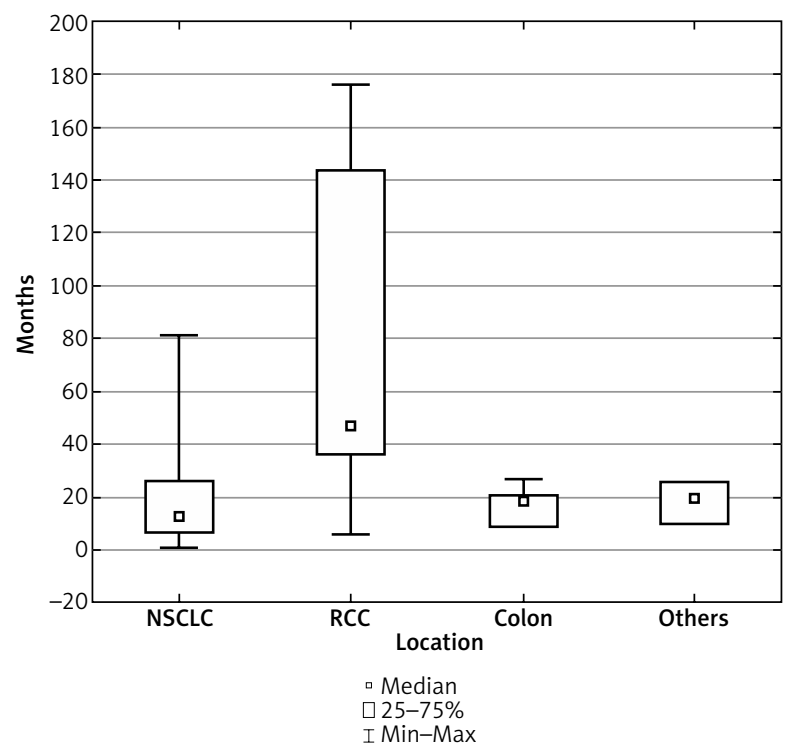

Fig. 1. Time from primary treatment to adrenalectomy

Time from primary treatment to adrenalectomy differed depending on the primary tumour and was the longest for RCC, and this difference was statistically significant ( $p=0.000362)$ (Fig. 1).

In the presented series median OS $(95 \% \mathrm{Cl})$ was 18 months (mean 22 months) (Fig. 2), and the 2-year actual OS rate was 43.6\% (17/39 pts).

Median DFS was 18 months (mean 20.7 months) and varied from 6 (others group) to 25.5 months (colorectal cancer group) although multivariate analysis did not show any independent significant factors for DFS $(p=0.089)$.

In the univariate analysis, a significant influence on OS was demonstrated for primary tumour type. Significantly better OS was observed for patients with colorectal cancer metastases (median 29.5 months) compared to NSCLC (median 10 months) and others (median 10 months) ( $p=0.0119$ ). In NSCLC patients no difference in median OS was found between the synchronic (median - 20, mean - 17.7 months) and metachronous (median - 20, mean - 22.4 months) group $(p=0.787)$. For RCC median OS was 24 months. None of the patients with melanoma, ovarian or urothelial cancer lived longer than 20 months. There was no statistically significant difference in survival depending on sex, age and metastatic tumour size. However, in the group of patients who died, the size of metastasis (mean $76.5 \mathrm{~mm}$ ) was significantly bigger than in the group of survivors (mean $52.5 \mathrm{~mm})(p=0.026)$.

\section{Discussion}

Resections of metastases to the adrenals started in the mid-1970s [14]. Then, after a study which showed significantly better survival in patients after resection of adrenal metastases from NSCLC and for those who were treated conservatively, it became an accepted treatment [15]. Later, many publications demonstrated good results of surgical treatment for patients with isolated metastases of different types of cancers in selected patients [16, 17].

There are few prospective data and a lack of randomized evidence to confirm that local therapy to the adrenal

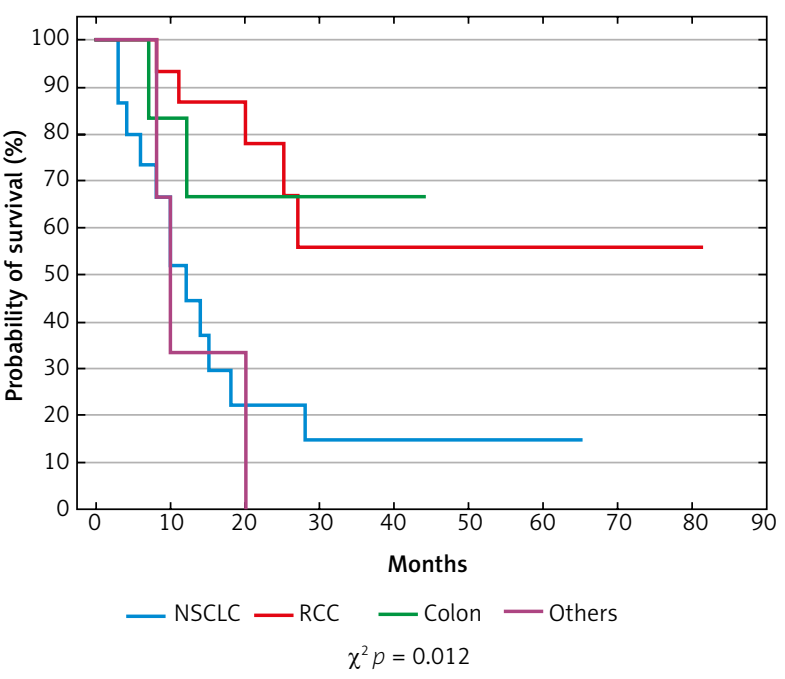

Fig. 2. Kaplan-Meier survival probability

metastases alters the natural course of disease. Treatment of patients with adrenal metastases varies among institutions and is strongly dependent on the clinic's discretion.

In our consecutive 14-year series, we analysed 39 patients treated with adrenalectomy for adrenal metastases of solid tumours. The number of patients is similar to previously reported series $[18,19]$. It is worth noting that even in multicentre studies the number of analysed patients rarely exceeds several dozen cases [9, 20,21].

In the presented group, the predominant cancers which metastasised to adrenals were NSCLC and RCC, which together corresponds to over $74 \%$ of metastases. On pooling data, the three most common primary histologies were lung cancer (small and non-small), RCC and melanoma, representing $32 \%, 22 \%$ and $15 \%$ of patients respectively [22]. In another large series, most frequent primary tumours were lung $(46.6 \%)$, colorectal $(13.5 \%)$, renal $(11.7 \%)$, breast (3.5\%) and melanoma (3.5\%) [23]. The relative prevalence of each primary cancer varies according to the source of data and the geographic region. Data from Asia indicated a high prevalence of stomach (14\%), oesophagus (12\%) and liver/bile duct cancers (10\%) with a paucity of breast cancer and melanoma [4], but even in some European series liver and bile duct cancer may be the most frequent (29\%) primary tumour metastasizing to adrenals [24].

Mean DFl in the present series was 42 months with the longest interval for RCC (176 months), and these findings were very similar to previously published findings -39 and 156 months respectively [25]. These findings are worth taking into account in follow-up protocols.

In the present study, the median OS was 18 months and was superior to some studies with median survival of 14 months [26] and similar to other series with 23 months [18]. On the other hand, there are only a few studies with considerably better median survival of 29-32 months $[9,20]$.

Actual 2-year survival in the present material was $43.6 \%$. This is in line with other investigators, who report a 2-year survival rate of $31-40 \%[24,26]$; however, there are studies with better survival reaching $61 \%$ at 2 years [9]. 
In patients with adrenal metastases, tumour type was previously suggested to be a prognostic factor for better survival [27]. In the present series tumour type was a significant prognostic factor with the most favourable outcome for patients with colorectal cancer, opposite to the shortest survival observed in patients with NSCLC. These observations are similar to previous reports [18].

Primary renal cancer seems to have a good prognosis in comparison to other primary malignancies. In 41 patients, median OS was 14 months and 5-year survival was observed in $21 \%$, while for patients with primary renal cancer the 5-year survival rate was 50\% [24]. Significant differences in survival with regard to tumour type were seen in another report, with longer survival for patients with colorectal carcinoma or RCC and shorter for those with NSCLC or melanoma [18].

Findings of other studies are ambiguous regarding factors which determine survival after adrenalectomy for metastases. In a group of 90 patients with dominant primary melanoma and lung metastases and actual 5-year survival of $26.2 \%$, there was no statistically significant difference in OS for those who underwent adrenalectomy for metastases from lung cancer compared with those with melanoma or other tumours [28].

Not only renal primary but also a tumour size smaller than $5 \mathrm{~cm}$ in multivariate analysis were independently associated with longer survival in a series of 65 consecutive patients submitted to adrenalectomy for metastatic disease [25].

Analysis of the present series did not reveal that the median duration of survival depends on tumour size, and this finding is concordant with other reports [17, 29]. Opposite to this, there are studies which demonstrate that adrenal metastases with large diameter (> $45 \mathrm{~mm}$ ) are associated with a worse prognosis [18]. There are also series in which analysis of the entire group did not reveal that the median duration of survival depended on tumour size except in the colorectal cancer group where a significant difference in favour of the smaller tumours $(<60 \mathrm{~mm})$ was found [10].

Since the first report of laparoscopic adrenalectomy [30], it has become the gold standard of treatment for benign adrenal masses [31] and is gaining acceptance in treating metastatic adrenal lesions [10, 21, 32, 33].

All patients in the present material were operated on by open surgery. Laparoscopic adrenalectomy in our centre was introduced in 2013 and until 2017 was restricted to unsuspected adrenal tumours. Since 2018 we have started to perform laparoscopic adrenalectomy also in patients with suspected metastases, but they are not part of this analysis.

We are aware that the results of this study are burdened low numbers of patients in different groups, and statistical analysis in such small groups may be inconclusive.

It must be emphasized that the majority of studies, like the present one, are characterised by exceptionally varied study groups with a limited number of patients, which makes the results difficult to compare. Analyses of prognostic factors in case series are guided by the error of selection and bias and many variables are inter-related.
All observation concentrates on adrenalectomy alone (without a discussion on additional adjuvant systemic treatment after surgery, which may affect survival). Larger pooled analyses using standardised protocols and precise definitions are necessary to identify the optimal indications for adrenalectomy for adrenal metastasis.

\section{Conclusions}

Our study confirms that adrenalectomy is associated with a good survival rate with minimal perioperative morbidity in selected patients. Factors associated with patient survival included tumour type, and the longest median survival was found for colorectal cancer metastases.

The authors declare no conflict of interest.

\section{References}

1. Mansmann G, Lau J, Balk E, Rotberg M, Miyachi Y, Bornstein SR. The clinically inapparent adrenal mass: update in diagnosis and management. Endocr Rev 2004; 25: 309-340.

2. Bullock WK, Hirst AE Jr. Metastatic carcinoma of the adrenal gland Am J Med Sci 1953; 226: 521-524.

3. Abrams HL, Spiro R, Goldstein N. Metastases in carcinoma; analysis of 1000 autopsied cases. Cancer 1950; 3: 74-85.

4. Lam KY, Lo CY. Metastatic tumours of the adrenal glands: a 30-year experience in a teaching hospital. Clin Endocrinol (Oxf) 2002; 56: 95-101.

5. Young WF Jr. Clinical practice. The incidentally discovered adrenal mass. N Engl J Med 2007; 356: 601-610.

6. Blake MA, Cronin CG, Boland GW. Adrenal imaging. AJR Am J Roentgenol 2010; 194: 1450-1460.

7. Lattin GE Jr, Sturgill ED, Tujo CA, Marko J, Sanchez-Maldonado KW, Craig WD, Lack EE. From the radiologic pathology archives: Adrenal tumours and tumour-like conditions in the adult: radiologic-pathologic correlation. Radiographics 2014; 34: 805-829.

8. Grossman A, Koren R, Tirosh A, et al. Prevalence and clinical characteristics of adrenal incidentalomas in potential kidney donors. Endocr Res 2016; 41: 98-102.

9. Moreno P, de la Quintana Basarrate A, Musholt TJ, et al. Adrenalectomy for solid tumor metastases: results of a multicenter European study. Surgery 2013; 154: 1215-1222; discussion 1222-1223.

10. Marangos IP, Kazaryan AM, Rosseland AR, et al. Should we use laparoscopic adrenalectomy for metastases? Scandinavian multicenter study. J Surg Oncol 2009; 100: 43-47.

11. Gryn A, Peyronnet B, Manunta A, et al. Patient selection for laparoscopic excision of adrenal metastases: A multicenter cohort study. Int J Surg 2015; 24 (Pt A): 75-80.

12. Mittendorf EA, Lim SJ, Schacherer CW, et al. Melanoma adrenal metastasis: natural history and surgical management. Am J Surg 2008; 195: 363-368; discussion 368-369.

13. Vazquez BJ, Richards ML, Lohse CM, Thompson GB, Farley DR, Grant CS, Huebner M, Moreno J. Adrenalectomy improves outcomes of selected patients with metastatic carcinoma. World J Surg 2012; 36: 1400-1405.

14. Yonemoto RH, Tan MS, Byron RL, Riihimaki DU, Keating J, Jacobs W. Randomized sequential hormonal therapy vs adrenalectomy for metastatic breast carcinoma. Cancer 1977; 39: 547-555.

15. Luketich JD, Burt ME. Does resection of adrenal metastases from non-small cell lung cancer improve survival? Ann Thorac Surg 1996; 62: 1614-1616.

16. Branum GD, Epstein RE, Leight GS, Seigler HF. The role of resection in the management of melanoma metastatic to the adrenal gland. Surgery 1991; 109: 127-131. 
17. Sarela Al, Murphy I, Coit DG, Conlon KC. Metastasis to the adrenal gland: the emerging role of laparoscopic surgery. Ann Surg Oncol 2003; 10: 1191-1196.

18. Muth A, Persson F, Jansson S, Johanson V, Ahlman H, Wängberg B. Prognostic factors for survival after surgery for adrenal metastasis. Eur J Surg Oncol 2010; 36: 699-704.

19. Zheng QY, Zhang GH, Zhang Y, Guo YL. Adrenalectomy may increase survival of patients with adrenal metastases. Oncol Lett 2012; 3: 917-920.

20. Valeri A, Bergamini C, Tozzi F, Martellucci J, Di Costanzo F, Antonuz zo L. A multi-center study on the surgical management of metastatic disease to adrenal glands. J Surg Oncol 2011; 103: 400-405.

21. Goto T, Inoue T, Kobayashi T, et al. Feasibility of laparoscopic adrenalectomy for metastatic adrenal tumors in selected patients: a retrospective multicenter study of Japanese populations. Int J Clin Oncol 2020; 25: 126-134.

22. Gunjur A, Duong C, Ball D, Siva S. Surgical and ablative therapies for the management of adrenal 'oligometastases' - A systematic review. Cancer Treat Rev 2014; 40: 838-846.

23. Duh QY. Resecting isolated adrenal metastasis: why and how? Ann Surg Oncol 2003; 10: 1138-1139.

24. Hornstein I, Schwarz C, Ebbing S, Hoppe-Lotichius M, Otto G, Lang $H$, Musholt TJ. Surgical resection of metastases to the adrenal gland: a single center experience. Langenbecks Arch Surg 2015; 400: 333-339.

25. Zerrweck C, Caiazzo R, Clerquin B, et al. Renal origin and size are independent predictors of survival after surgery for adrenal metastasis. Ann Surg Oncol 2012; 19: 3621-3626.

26. Strong VE, D’Angelica M, Tang L, et al. Laparoscopic adrenalectomy for isolated adrenal metastasis. Ann Surg Oncol 2007; 14: 3392 3400.

27. Lo CY, van Heerden JA, Soreide JA, Grant CS, Thompson GB, Lloyd RV, Harmsen WS. Adrenalectomy for metastatic disease to the adrenal glands. Br J Surg 1996; 83: 528-531.

28. Romero Arenas MA, Sui D, Grubbs EG, Lee JE, Perrier ND. Adrenal metastectomy is safe in selected patients. World J Surg 2014; 38: 1336-1342.

29. Sebag F, Calzolari F, Harding J, Sierra M, Palazzo FF, Henry JF. Isolated adrenal metastasis: the role of laparoscopic surgery. World J Surg 2006; 30: 888-892.

30. Gagner M, Lacroix A, Bolté E. Laparoscopic adrenalectomy in Cushing's syndrome and pheochromocytoma. N Engl J Med 1992; 327: 1033

31. Hansen P, Bax T, Swanstrom L. Laparoscopic adrenalectomy: his tory, indications, and current techniques for a minimally invasive approach to adrenal pathology. Endoscopy 1997; 29: 309-314.

32. Sarela AI, Murphy I, Coit DG, Conlon KC. Metastasis to the adrenal gland: the emerging role of laparoscopic surgery. Ann Surg Onco 2003; 10: 1191-1196.

33. Ma X, Li H, Zhang X, et al. Modified anatomical retroperitoneoscopic adrenalectomy for adrenal metastatic tumor: technique and survival analysis. Surg Endosc 2013; 27: 992-999.

\section{Address for correspondence}

\section{Radosław Samsel}

Department of Surgery

Clinic of Oncological Surgery and Neuroendocrine Tumors

Maria Sklodowska-Curie Institute - Oncology Center

15 Wawelska St.

02-034, Warsaw, Poland

e-mail: radeksamsel@o2.pl

Submitted: 2.12 .2019

Accepted: 26.01 .2020 